\title{
The effect of empirical superior vena cava isolation during total thoracoscopic ablation in patients with persistent atrial fibrillation
}

\author{
Hee-jin Kwon ${ }^{1}$, Dong Seop Jeong ${ }^{2}$, Hye Ree Kim², Seung-Jung Park², Kyoung-Min Park ${ }^{2}$, \\ june soo kim², and Young Keun $\mathrm{On}^{2}$ \\ ${ }^{1}$ Chungnam National University Hospital \\ ${ }^{2}$ Samsung Medical Center
}

September 29, 2021

\begin{abstract}
Introduction: In patients with non-paroxysmal AF, various ablation strategies have been attempted to target non-pulmonary vein (PV) foci or to achieve substrate modification beyond pulmonary vein isolation (PVI). The efficacy of empirical ablation of the SVC, one of the most common non-PV foci, is unclear. The aim of this study was to investigate the efficacy and safety of additional superior vena cava (SVC) isolation in patients with non-paroxysmal atrial fibrillation (AF) undergoing thoracoscopic surgical ablation. Methods and Results: A total of 191 patients with persistent or longstanding persistent AF was enrolled. All patients underwent total thoracoscopic surgical ablation for AF, and half of them also received empirical SVC isolation. We compared the atrial-tachyarrhythmia (ATa)-free survival rate and procedure-related complications in the two groups of patients. The 3-year ATa-free survival rate was $53 \%$ in the SVC-isolation group and $52 \%$ in the no-SVC-isolation group, ( $\mathrm{p}=0.644)$. There were no differences between the two groups with respect to AF type or LA size. Procedure-related complications occurred in 12 patients (6\%). Pacemakers were implanted only in 3 patients from the SVC-isolation group. The only factor influencing recurrence of ATa was LA diameter. Conclusions: Empirical SVC isolation during thoracoscopic ablation for persistent AF did not improve patient outcomes.
\end{abstract}

The effect of empirical superior vena cava isolation during total thoracoscopic ablation in patients with persistent atrial fibrillation

Running title: Empirical SVC isolation during TTA

Hee-Jin Kwon $\mathrm{MD}^{1 *}$, Dong Seop Jeong MD $\mathrm{PhD}^{2 *}$, Hye-Ree Kim MD PhD ${ }^{3}$, Seung-Jung Park MD PhD ${ }^{3}$, Kyoung-Min Park MD $\mathrm{PhD}^{3}$, June Soo Kim MD $\mathrm{PhD}^{3}$, Young Keun On MD $\mathrm{PhD}^{3}$

${ }^{1}$ Division of Cardiology, Department of Internal Medicine, Chungnam National University Hospital, Chungnam National University School of Medicine, Daejeon, Republic of Korea

${ }^{2}$ Department of Thoracic and Cardiovascular Surgery, Heart Vascular and Stroke Institute, Samsung Medical Center, Sungkyunkwan University School of Medicine, Seoul, Republic of Korea

${ }^{3}$ Division of Cardiology, Department of Internal Medicine, Heart Vascular and Stroke Institute, Samsung Medical Center, Sungkyunkwan University School of Medicine, Seoul, Republic of Korea

* The first two authors contributed equally to this work.

Acknowledgement: None

Disclosure: None

Funding: None 


\title{
Address for correspondence:
}

Young Keun On, MD, PhD, FHRS

Division of Cardiology, Department of Internal Medicine, Heart Vascular and Stroke Institute, Samsung Medical Center, Sungkyunkwan University School of Medicine

81 Irwon-ro, Gangnam-gu, Seoul 06351, Republic of Korea

Tel: +82-2-3410-3419, Fax: +82-2-3410-3849

E-mail: yk.on@samsung.com

\begin{abstract}
Introduction: In patients with non-paroxysmal AF, various ablation strategies have been attempted to target non-pulmonary vein (PV) foci or to achieve substrate modification beyond pulmonary vein isolation (PVI). The efficacy of empirical ablation of the SVC, one of the most common non-PV foci, is unclear. The aim of this study was to investigate the efficacy and safety of additional superior vena cava (SVC) isolation in patients with non-paroxysmal atrial fibrillation $(\mathrm{AF})$ undergoing thoracoscopic surgical ablation.
\end{abstract}

Methods and Results: A total of 191 patients with persistent or longstanding persistent AF was enrolled. All patients underwent total thoracoscopic surgical ablation for AF, and half of them also received empirical SVC isolation. We compared the atrial-tachyarrhythmia (ATa)-free survival rate and procedure-related complications in the two groups of patients. The 3-year ATa-free survival rate was $53 \%$ in the SVC-isolation group and $52 \%$ in the no-SVC-isolation group, $(\mathrm{p}=0.644)$. There were no differences between the two groups with respect to AF type or LA size. Procedure-related complications occurred in 12 patients (6\%). Pacemakers were implanted only in 3 patients from the SVC-isolation group. The only factor influencing recurrence of ATa was LA diameter.

Conclusions: Empirical SVC isolation during thoracoscopic ablation for persistent AF did not improve patient outcomes.

Keywords persistent atrial fibrillation; thoracoscopic surgical ablation; superior vena cava; empirical isolation; non-PV trigger

\section{Introduction}

Pulmonary vein isolation (PVI) is the cornerstone of atrial fibrillation (AF) treatment ${ }^{1}$; however, despite complete PVI, some patients experience recurrence of AF and of non-paroxysmal AF, in particular ${ }^{2,3}$. Many investigators have postulated that both substrate modification and trigger isolation are necessary for effective treatment of persistent or longstanding AF, and various ablation strategies, such as non-PV trigger, linear line, complex fractionated atrial electrogram (CFAE), or rotor ablation have been assessed ${ }^{4,5}$.

Non-PV trigger sources are present in approximately $11 \%$ of patients with persistent and long-standing persistent $\mathrm{AF}{ }^{6,7}$. The superior vena cava (SVC) is one of the most common non-PV AF trigger sites, along with the coronary sinus, vein of Marshall, left atrial posterior wall, interatrial septum, and crista terminalis. The SVC acts as both an initiator and perpetuator of $\mathrm{AF}^{1,8}$. Previous studies have shown that arrhythmogenic SVC or empirical SVC isolation in combination with PVI improves clinical outcomes ${ }^{9-11}$; however, the efficacy of SVC isolation in patients with persistent or chronic atrial fibrillation remains controversial ${ }^{9}$.

Thoracoscopic surgical ablation has been advanced as an alternative for non-paroxysmal AF patients. This approach allows durable PVI through a direct epicardial approach, with the added benefit of ablating epicardial structures such as the ganglion plexus (GP) or Marshall's ligament, while excluding the left atrial appendage and limiting the risk of phrenic nerve injury. To date, there have been no studies assessing the efficacy and safety of additional empirical SVC isolation in thoracoscopic surgical ablation for AF.

The aim of this study was to investigate the effect of empirical SVC isolation in thoracoscopic surgical ablation of patients with non-paroxysmal AF. 


\section{Methods}

\section{Study population}

From January 2012 to December 2018, consecutive patients who underwent thoracoscopic surgical ablation for persistent or longstanding persistent AF were analyzed retrospectively. Patients with prior history of surgical or catheter ablation or in whom previous complete thoracoscopic surgical ablation had failed due to thoracic adhesions were excluded. In addition, patients who underwent a hybrid approach, in which endocardial catheter ablation was completed immediately after surgical ablation, were also excluded. Half of the enrolled patients received empirical SVC isolation, and patient outcomes in the SVC-isolation and no-SVC-isolation groups were compared. All data were collected through a review of medical records. This study was approved by the Institutional Review Board of Samsung Medical Center, and informed consent was waived (IRB No. 2020-06-159).

\section{Surgical procedures}

Total thoracoscopic ablation (TTA) refers to a video-assisted thoracoscopic surgical ablation technique using bipolar radiofrequency energy without the assistance of the Da Vinci system (Intuitive Surgical, Sunnyvale, CA, USA) or cardiopulmonary bypass. First, three ports were introduced on the right side. One $5 \mathrm{~mm}$ port was inserted into the fourth intercostal space, and CO gas was injected to expand the chest cavity and secure the surgical field. The remaining two ports were placed in the third intercostal space at the anterior axillary line and the sixth intercostal space at the midaxillary line. After pericardial tenting, a lighted dissector (AtriCure ${ }^{\circledR}$ Lumitip, Atricure, Inc., Cincinnati, OH, USA) was used to pass a rubber band under the antrum of the pulmonary vein (PV) through the oblique sinus. An AtriCure ${ }^{\circledR}$ Isolator ${ }^{\circledR}$ transpolar clamp was positioned around the PV antrum through a connection to the rubber band. Bipolar radiofrequency energy was delivered to the clamp six times to achieve PV isolation. Superior and inferior lines connecting the PV isolation lines were created using a linear pen device (MLP, Atricure, Inc.). Ganglionated plexuses were subsequently ablated with bipolar energy under high-frequency pacing. The procedure was performed in the same way on the left side. The endpoint of each ablation is the confirmation of bidirectional acute conduction block across pulmonary veins isolation defined as the absence of sensed atrial potentials in the $\mathrm{PVs}$ and pacing conduction to the atria from PVs in patients in sinus rhythm. A high-frequency stimulation is used at ablation and its response is defined as [?]50\% increase in the R-R interval. Using a bipolar ablation pen (Isolator Transpolar pen), the high-frequency stimulation is delivered (cycle length $60 \mathrm{~ms}, 16 \mathrm{~Hz}$, pulse width $1.0 \mathrm{~ms}$ ) with output increments from 1 to $25 \mathrm{~mA}$. When the high-frequency stimulation does not evoke a vagal response, ablation is performed on the basis of anatomic landmarks. After PV and ganglionated plexus ablation, the ligament of Marshall was dissected and ablated. Once ablations were completed and conduction block was confirmed, the left atrial auricle was removed with an endoscopic stapling device. In patients in the SVC group, a clamp was placed above the SVC and RA junctions, and ablation was performed using the bipolar clamp previously used for pulmonary vein isolation. Circular ablation of the SVC was performed twice following an exit block test (Supplementary Figure S1). All procedures were performed by one experienced cardiac surgeon (DSJ).

\section{Follow up}

After surgery, all patients were monitored in the intensive care unit for the first 24 hours. After confirming that there was no bleeding or pericardial effusion, heparin infusion was started 4 hours after the operation; on the day after the operation, the heparin infusion was discontinued and the patient was switched to oral anticoagulation (non-vitamin $\mathrm{K}$ antagonist or warfarin). Anti-arrhythmic drugs were continued after surgery. All patients had follow-up evaluations at 2 weeks, 3 months and 6 months postoperatively and every 6 months thereafter. At every visit, 12-lead electrocardiography (ECG) and 24-hour Holter monitoring were performed. After a 3-month blanking period, recurrence was defined as the detection of any atrial tachyarrhythmias (ATa) including AF, atrial flutter (AFL) or atrial tachycardia (AT) occurring for a duration of more than 30 seconds. Cardioversion was performed as needed during the blanking period. In patients with recurrence of ATa, additional interventions, such as antiarrhythmic drugs, catheter ablation, or DC 
cardioversion, were performed in consideration of the patient's status. In general, antiarrhythmic drugs (AADs) were discontinued after 3 months if there was no evidence of recurrence. Oral anticoagulants (OACs) were continued postoperatively; in patients with no history of stroke, low $\mathrm{CHA}_{2} \mathrm{DS}_{2}$-VASc score, and wellmaintained sinus rhythm, OACs were discontinued on the decision of the attending physician. During all follow-up periods, complications were defined as events requiring additional interventions, medications, or that lengthened the duration of hospital stay.

\section{Statistical analyses}

Categorical variables are reported as numbers and percentages, while continuous variables are presented as the mean + - standard deviation (SD). For comparison between the two groups based on SVC isolation, a chi-square test or Fisher's exact test was used for categorical variables, and an independent sample T-test was used for continuous variables. The ATa-free survival rate was analyzed using the Kaplan-Meier method, and the survival rates of the two groups were compared using a log-rank test. Factors associated with ATa recurrence were identified using univariate and stepwise multivariate analyses in Cox regression. All variables with $\mathrm{p}$ values $<0.10$ in the univariate analyses were entered into the multivariate analyses. A two-sided $\mathrm{P}$-value $<0.05$ was considered statistically significant. All statistical analyses were conducted using $\operatorname{SPSS}^{(\mathrm{r})}$ software (Version 23.0; IBM, Armonk, NY).

\section{Results}

\section{Baseline patient characteristics}

Of the 191 total patients, 98 (51\%) received SVC isolation (SVC isolation group), and 93 (49\%) did not (no-SVC isolation group). The baseline patient characteristics are presented in Table 1. The mean age was $57+-8$ years, and $89 \%$ were male. There were no significant difference in comorbidities between the two groups. The mean AF duration was $44+-44$ months. Persistent AF and longstanding persistent AF accounted for $55 \%$ and $45 \%$ of the patient diagnoses, respectively. The SVC-isolation group contained a significantly greater percentage of patients with longstanding persistent AF (53\%) than did the no-SVCisolation group $(36 \%, \mathrm{p}=0.015)$, and the LA size was larger (LA diameter $49+-6$ vs. $46+-7 \mathrm{~mm}, \mathrm{p}=0.002$ ). The mean left ventricular ejection fraction was $59+-8 \%$.

\section{Surgical procedure and complications}

All patients underwent PVI. More than $90 \%$ received roof and posterior line ablation or ganglion plexus (GP) ablation, and there were no significant differences between the two groups with respect to the use of these procedures. Twenty-one patients (11\%) also underwent additional linear ablation from the SVC to IVC. Marshall ligament division was performed in a lower percentage of patients in the no-SVC- than in the SVC-isolation group. Left atrial appendage (LAA) removal was performed in all but two patients (Table 2).

Procedure-related complications occurred in 12 patients $(6 \%)$, and there were no differences between the two groups ( $7 \%$ vs. $6 \%, \mathrm{p}=0.925)$. There were no patients with phrenic nerve injury in either group. Pacemakers were implanted only in 3 patients from the SVC-isolation group: two of these patients had preexisting sinus node dysfunction before ablation, and the other had a newly discovered arrhythmia after the procedure and had a pacemaker implanted. One patient died of an unknown cause one month after surgical ablation (Table 3).

\section{Recurrence of atrial tachyarrhythmia}

The overall mean follow-up duration was 32+-17 months. The 1-year and 3-year ATa-free survival rates were $68 \%$ and $53 \%$ in the SVC-isolation group and $75 \%$ and $52 \%$ in the no-SVC-isolation group, respectively, and there were no significant differences between the two groups $(\mathrm{p}=0.644$, Figure 1$)$. There were also no differences according to AF type: no ATa recurrence was reported in $62 \%$ and $67 \%(\mathrm{p}=0.207)$ of patients with persistent $\mathrm{AF}$ and $43 \%$ and $38 \%(\mathrm{p}=0.211$ ) of those with longstanding persistent AF in the SVC isolation and control groups, respectively (Figures 2A, 2B). There were also no differences in ATa-free survival according to LA diameter (Figures $2 \mathrm{C}, 2 \mathrm{D}$ ). Table 4 presents the results of the univariate and multivariate analyses to 
identify determinants of ATa recurrence. The only factor influencing the recurrence of ATa was LA diameter in the multivariate analyses (hazard ratio 1.079, 95\% confidence interval $1.040-1.121, \mathrm{p}<0.001$ ).

\section{Discussion}

In this study, we investigated the efficacy and safety of additional SVC isolation when performing thoracoscopic surgical ablation in patients with non-paroxysmal AF. To our knowledge, this is the first study to evaluate the efficacy and safety of additional SVC isolation in surgical ablation for patients with AF. Our study demonstrated that empirical SVC isolation did not improve outcomes in patients with non-paroxysmal AF. A larger LA size was the only independent predictor of ATa recurrence, which was consistent with the results of previous studies. Complications occurred in $6 \%$ of patients. There were no patients with phrenic nerve injury, but three patients in the SVC-isolation group required pacemaker implantation.

The SVC is one of the most common non-PV AF trigger sites. The SVC has been shown to act not only as an initiator, but also as a perpetuator of $\mathrm{AF}^{8,12}$. During embryogenesis, the SVC originates from the sinus venosus, which is also the origin of the sinoatrial node ${ }^{13}$. This can explain the arrhythmogenicity of this structure. There have been many studies of SVC isolation using catheter ablation. Additional SVC isolation with PVI has been shown to improve outcomes in AF, even with documented SVC triggers or an empirical strategy ${ }^{9,11,14,15}$ : Arruda et al. reported lower AF recurrence with empirical SVC isolation 14; similar results were obtained in a prospective randomized trial assessing patients with paroxysmal and non-paroxysmal $\mathrm{AF}^{9}$. However, although empirical SVC isolation improved outcomes in paroxysmal AF ( $77 \%$ vs. $90 \%, \mathrm{p}=0.04)$, outcomes in patients with persistent $\mathrm{AF}$ were not significantly improved, consistent with the results of our study using thoracoscopic surgical ablation in patients with non-paroxysmal AF.

$\mathrm{Xu}$ et al. investigated the role of SVC in patients with long-standing persistent AF who underwent extensive endocardial ablation ${ }^{16}$. The arrhythmogenicity of the SVC was confirmed in only 1 of the patients $(0.98 \%)$. Both AF duration and LA diameter were determinants of AF recurrence. Similarly, in another study, the prevalence of arrhythmogenic SVC in persistent and longstanding persistent AF was very rare, at $1.9 \%$ and $1.3 \%$, respectively ${ }^{17}$. Small LA size and typical atrial flutter were reported to be independent predictors of arrhythmogenic SVC. Atrial fibrillation is a progressive disease characterized by chronic atrial structural remodeling, including cellular hypertrophy, fibroblast proliferation, and tissue fibrosis. Left atrial enlargement is a surrogate marker of elevated left ventricular filling pressure and the result of atrial remodeling. These contradictory results suggest that the mechanism of AF in patients with arrhythmogenic SVC differs from the typical course of AF progression.

In the present study, we found an ATa-free survival rate of $67 \%$ at 12 months for non-paroxysmal AF patients who underwent thoracoscopic surgical ablation. This outcome was better than that reported in a previous study in which catheter ablation was performed on patients with similar LA diameter, an independent predictor of AF recurrence (43\% ATa-free survival at 12 months). These findings suggest that epicardial ablation is an alternative treatment option for patients with advanced AF with larger LA size. Empirical SVC ablation did not provide any additional benefit with respect to patient outcomes.

\section{Limitations}

Our study has several limitations. This study was retrospective in design and reports the findings from a single center. Since epicardial ablation was performed using a thoracoscopic approach, the arrhythmogenicity of SVC could not be evaluated. In addition, the ATa recurrence rate might have been underestimated when using 12-lead ECG or Holter ECG rather than continuous monitoring. Further larger and randomized studies are required to support our results.

\section{Conclusions}

Empirical SVC isolation during thoracoscopic ablation for persistent AF was safe. Empirical SVC isolation in persistent AF patients did not improve outcomes and had no additional benefit; however, further larger studies are needed to confirm this finding. 
Conflict of interest None declared

\section{Acknowledgement None}

\section{References}

1. Haissaguerre M, Jais P, Shah DC, Takahashi A, Hocini M, Quiniou G, Garrigue S, Le Mouroux A, Le Metayer P, Clementy JJNEJoM: Spontaneous initiation of atrial fibrillation by ectopic beats originating in the pulmonary veins 1998;339:659-666.

2. Oral H, Pappone C, Chugh A, Good E, Bogun F, Pelosi Jr F, Bates ER, Lehmann MH, Vicedomini G, Augello GJNEJoM: Circumferential pulmonary-vein ablation for chronic atrial fibrillation 2006;354:934-941.

3. Haissaguerre M, Hocini M, Sanders P, Sacher F, Rotter M, Takahashi Y, Rostock T, HSU LF, Bordachar P, Reuter SJJoce: Catheter ablation of long-lasting persistent atrial fibrillation: Clinical outcome and mechanisms of subsequent arrhythmias 2005;16:1138-1147.

4. Nademanee K, McKenzie J, Kosar E, Schwab M, Sunsaneewitayakul B, Vasavakul T, Khunnawat C, Ngarmukos TJJotACoC: A new approach for catheter ablation of atrial fibrillation: mapping of the electrophysiologic substrate 2004;43:2044-2053.

5. Narayan SM, Krummen DE, Shivkumar K, Clopton P, Rappel W-J, Miller JMJJotACoC: Treatment of atrial fibrillation by the ablation of localized sources: CONFIRM (Conventional Ablation for Atrial Fibrillation With or Without Focal Impulse and Rotor Modulation) trial 2012;60:628-636.

6. Santangeli P, Zado ES, Hutchinson MD, Riley MP, Lin D, Frankel DS, Supple GE, Garcia FC, Dixit S, Callans DJJHR: Prevalence and distribution of focal triggers in persistent and long-standing persistent atrial fibrillation 2016;13:374-382.

7. Gokoğlan Y, Mohanty S, Güneş MF, Trivedi C, Santangeli P, Gianni C, Asfour IK, Bai R, Burkhardt JD, Horton RJCA, Electrophysiology: Pulmonary vein antrum isolation in patients with paroxysmal atrial fibrillation: more than a decade of follow-up 2016;9:e003660.

8. Miyazaki S, Takigawa M, Kusa S, Kuwahara T, Taniguchi H, Okubo K, Nakamura H, Hachiya H, Hirao K, Takahashi AJJoce: Role of arrhythmogenic superior vena cava on atrial fibrillation 2014;25:380-386.

9. Corrado A, Bonso A, Madalosso M, Rossillo A, Themistoclakis S, Di Biase L, Natale A, Raviele AJJoce: Impact of systematic isolation of superior vena cava in addition to pulmonary vein antrum isolation on the outcome of paroxysmal, persistent, and permanent atrial fibrillation ablation: results from a randomized study 2010;21:1-5.

10. Overeinder I, Osório TG, Călburean P-A, Bisignani A, Bala G, Sieira J, Ströker E, Al Houssari M, Mojica J, Boveda SJJoICE: Comparison between superior vena cava ablation in addition to pulmonary vein isolation and standard pulmonary vein isolation in patients with paroxysmal atrial fibrillation with the cryoballoon technique 2021:1-8.

11. Ejima K, Kato K, Iwanami Y, Henmi R, Yagishita D, Manaka T, Fukushima K, Arai K, Ashihara K, Shoda MJTAjoc: Impact of an empiric isolation of the superior vena cava in addition to circumferential pulmonary vein isolation on the outcome of paroxysmal atrial fibrillation ablation 2015;116:1711-1716.

12. Higuchi K, Yamauchi Y, Hirao K, Sasaki T, Hachiya H, Sekiguchi Y, Nitta J, Isobe MJHR: Superior vena cava as initiator of atrial fibrillation: factors related to its arrhythmogenicity 2010;7:1186-1191.

13. Gianni C, Sanchez JE, Mohanty S, Trivedi C, Della Rocca DG, Al-Ahmad A, Burkhardt JD, Gallinghouse GJ, Hranitzky PM, Horton RPJEE: Isolation of the superior vena cava from the right atrial posterior wall: a novel ablation approach 2018;20:e124-e132.

14. Arruda M, Mlcochova H, Prasad SK, Kilicaslan F, Saliba W, Patel D, Fahmy T, Morales LS, Schweikert $\mathrm{R}$, Martin DJJoce: Electrical isolation of the superior vena cava: an adjunctive strategy to pulmonary vein 
antrum isolation improving the outcome of AF ablation 2007;18:1261-1266.

15. Zhang T, Wang Y, Liang Z, Zhao H, Han Z, Wang Y, Wu Y, Ren XJTAjoc: Effect of combined pulmonary vein and superior vena cava isolation on the outcome of second catheter ablation for paroxysmal atrial fibrillation 2020;125:1845-1850.

16. Xu K, Wang Y, Wu S, Zhou L, Zhao L, Jiang W, Zhang X, Liu XJEE: The role of superior vena cava in catheter ablation of long-standing persistent atrial fibrillation 2017;19:1670-1675.

17. Miyazaki S, Taniguchi H, Kusa S, Ichihara N, Nakamura H, Hachiya H, Iesaka YJHR: Factors predicting an arrhythmogenic superior vena cava in atrial fibrillation ablation: insight into the mechanism 2014;11:15601566 .

Table 1 Baseline Characteristics

\begin{tabular}{|c|c|c|c|c|}
\hline Variables & Total $(n=191)$ & $\begin{array}{l}\text { No SVC ablation } \\
(\mathrm{n}=93,49 \%)\end{array}$ & $\begin{array}{l}\text { SVC ablation } \\
(\mathrm{n}=98,51 \%)\end{array}$ & p-value \\
\hline Gender (Female) & $21(11 \%)$ & $15(16 \%)$ & $6(6 \%)$ & 0.025 \\
\hline Age (year) & $57.1 \pm 8.2$ & $56.7 \pm 9.3$ & $57.4 \pm 7.0$ & 0.542 \\
\hline $\begin{array}{l}\text { Body mass index } \\
\left(\mathrm{kg} / \mathrm{m}^{2}\right)\end{array}$ & $26.0 \pm 3.4$ & $25.9 \pm 3.8$ & $26.1 \pm 3.0$ & 0.561 \\
\hline \multicolumn{5}{|l|}{ Comorbidities } \\
\hline Hypertension & $81(42 \%)$ & $36(39 \%)$ & $45(46 \%)$ & 0.314 \\
\hline Diabetes mellitus & $22(12 \%)$ & $11(12 \%)$ & $11(11 \%)$ & 0.896 \\
\hline Prior stroke/TIA & $24(13 \%)$ & $16(17 \%)$ & $8(8 \%)$ & 0.060 \\
\hline Heart failure & $26(14 \%)$ & $11(12 \%)$ & $15(16 \%)$ & 0.485 \\
\hline $\begin{array}{l}\text { Coronary artery } \\
\text { disease }\end{array}$ & $6(3 \%)$ & $1(1 \%)$ & $5(5 \%)$ & 0.118 \\
\hline $\begin{array}{l}\mathrm{CHA}_{2} \mathrm{DS}_{2} \mathrm{VASc} \\
\text { score }\end{array}$ & $1.3 \pm 1.3$ & $1.5 \pm 1.5$ & $1.1 \pm 1.1$ & 0.033 \\
\hline $\begin{array}{l}\text { AF duration } \\
\text { (month) }\end{array}$ & $44.4 \pm 44.2$ & $40.1 \pm 43.6$ & $48.4 \pm 44.6$ & 0.193 \\
\hline Type of AF & & & & 0.015 \\
\hline Persistent & $106(56 \%)$ & $60(64 \%)$ & $46(47 \%)$ & \\
\hline $\begin{array}{l}\text { Longstanding } \\
\text { persistent } \\
\text { Echocardiographic } \\
\text { data }\end{array}$ & $85(45 \%)$ & $33(36 \%)$ & $52(53 \%)$ & \\
\hline LV EF (\%) & $58.9 \pm 7.7$ & $58.2 \pm 8.4$ & $59.6 \pm 6.9$ & 0.210 \\
\hline $\begin{array}{l}\text { LA diameter } \\
(\mathrm{mm})\end{array}$ & $47.3 \pm 6.5$ & $45.8 \pm 6.7$ & $48.7 \pm 6.0$ & 0.002 \\
\hline $\begin{array}{l}\text { LA volume index } \\
\left(\mathrm{ml} / \mathrm{m}^{2}\right)\end{array}$ & $52.2 \pm 16.9$ & $48.6 \pm 6.0$ & $55.5 \pm 18.0$ & 0.005 \\
\hline
\end{tabular}

$\mathrm{AF}=$ atrial fibrillation; $\mathrm{EF}=$ ejection fraction; $\mathrm{LA}=$ left atrium; $\mathrm{SVC}=$ superior vena cava; $\mathrm{TIA}=$ transient ischemic attack

Table 2 Procedure Characteristics

\begin{tabular}{lllll}
\hline Variables & Total $(\mathbf{n}=\mathbf{1 9 1})$ & $\begin{array}{l}\text { No SVC ablation } \\
(\mathbf{n}=\mathbf{9 3}, \mathbf{4 9 \%})\end{array}$ & $\begin{array}{l}\text { SVC ablation } \\
(\mathbf{n}=\mathbf{9 8}, \mathbf{5 1 \%})\end{array}$ & p- value \\
\hline $\begin{array}{l}\text { Pulmonary vein } \\
\text { isolation }\end{array}$ & $191(100 \%)$ & $93(100 \%)$ & $98(100 \%)$ & \\
\end{tabular}




\begin{tabular}{|c|c|c|c|c|}
\hline Variables & Total $(n=191)$ & $\begin{array}{l}\text { No SVC ablation } \\
(\mathrm{n}=93,49 \%)\end{array}$ & $\begin{array}{l}\text { SVC ablation } \\
(\mathrm{n}=98,51 \%)\end{array}$ & p- value \\
\hline Roof line ablation & $178(93 \%)$ & $85(91 \%)$ & $96(98 \%)$ & 0.053 \\
\hline $\begin{array}{l}\text { Posterior line } \\
\text { ablation }\end{array}$ & $189(99 \%)$ & $92(99 \%)$ & $98(100 \%)$ & 0.487 \\
\hline $\begin{array}{l}\text { Ganglion plexus } \\
\text { ablation }\end{array}$ & $171(90 \%)$ & $83(89 \%)$ & $88(90 \%)$ & 0.901 \\
\hline $\begin{array}{l}\text { SVC to IVC } \\
\text { linear ablation }\end{array}$ & $21(11 \%)$ & $7(8 \%)$ & $14(14 \%)$ & 0.136 \\
\hline $\begin{array}{l}\text { Ligament of } \\
\text { Marshall division }\end{array}$ & $184(96 \%)$ & $86(94 \%)$ & $98(100 \%)$ & 0.012 \\
\hline $\begin{array}{l}\text { Left atrial } \\
\text { appendage } \\
\text { removed }\end{array}$ & $189(99 \%)$ & $91(98 \%)$ & $98(100 \%)$ & 0.236 \\
\hline
\end{tabular}

$\mathrm{IVC}=$ inferior vena cava

Table 3 Procedure-related complications

\begin{tabular}{lllll}
\hline Variables & Total $(\mathbf{n = 1 9 1 )}$ & $\begin{array}{l}\text { No SVC ablation } \\
(\mathbf{n = 9 3 , 4 9 \% )}\end{array}$ & $\begin{array}{l}\text { SVC ablation } \\
(\mathbf{n = 9 8 , 5 1 \%})\end{array}$ & p-value \\
\hline Total & $12(6 \%)$ & $6(7 \%)$ & $6(6 \%)$ & 0.925 \\
Pericarditis & $5(3 \%)$ & $3(3 \%)$ & $2(2 \%)$ & 0.676 \\
$\begin{array}{l}\text { Pacemaker } \\
\text { implantation }\end{array}$ & $3(2 \%)$ & 0 & $3(3 \%)$ & 0.247 \\
$\begin{array}{l}\text { Stroke } \\
\text { Death }\end{array}$ & $3(2 \%)$ & $2(2 \%)$ & $1(1 \%)$ & 0.613 \\
\hline
\end{tabular}

Table 4 Determinant of atrial tachyarrhythmia recurrence

\begin{tabular}{llll}
\hline Univariate & Hazard ratio & $\mathbf{9 5 \%}$ CI & p-value \\
\hline Age & 1.022 & $0.995-1.051$ & 0.111 \\
Sex & 1.200 & $0.635-2.268$ & 0.575 \\
AF type & 1.657 & $1.071-2.562$ & 0.023 \\
AF duration & 1.004 & $0.999-1.009$ & 0.090 \\
Left atrial diameter & 1.085 & $1.047-1.124$ & $<0.001$ \\
Left ventricular ejection fraction & 0.991 & $0.964-1.019$ & 0.524 \\
BMI & 1.059 & $0.994-1.127$ & 0.077 \\
Hypertension & 1.028 & $0.662-1.597$ & 0.901 \\
Diabetes & 0.917 & $0.459-1.833$ & 0.917 \\
SVC isolation & 1.108 & $0.718-1.710$ & 0.644 \\
GP ablation & 1.214 & $0526-2.800$ & 0.649 \\
Multivariate & & & \\
AF type & 1.596 & $0.980-2.598$ & 0.060 \\
AF duration & 1.000 & $0.994-1.005$ & 0.972 \\
Left atrial diameter & 1.079 & $1.040-1.121$ & $<0.001$ \\
BMI & 1.009 & $0.942-1.081$ & 0.793 \\
\hline
\end{tabular}


All variables with $\mathrm{p}$ values $<0.10$ in univariate analysis were included in the multivariate model, $\mathrm{GP}=$ ganglion plexus; other abbreviations as in Table 1.

\section{Figure legends}

Figure 1 Lesion set of thoracoscopic surgical ablation

$\mathrm{CS}=$ coronary sinus; GP = ganglion plexus; IVC = inferior vena cava; LAA = left atrial appendage; LLPV $=$ left lower pulmonary vein; LoM = ligament of Marshall; LUPV = left upper pulmonary vein; PVI = pulmonary vein isolation; RLPV = right lower pulmonary vein; RUPV = right upper pulmonary vein; SVC $=$ superior vena cava;

Figure 2 Atrial tachyarrhythmia free surival rate

Figure 3 Atrial tachyarrhythmia free survival rate according to subgroup

A persistent atrial fibrillation

B longstanding persistent atrial fibrillation

C left atrial diameter $<50 \mathrm{~mm}$

D left atrial diameter [?] $50 \mathrm{~mm}$

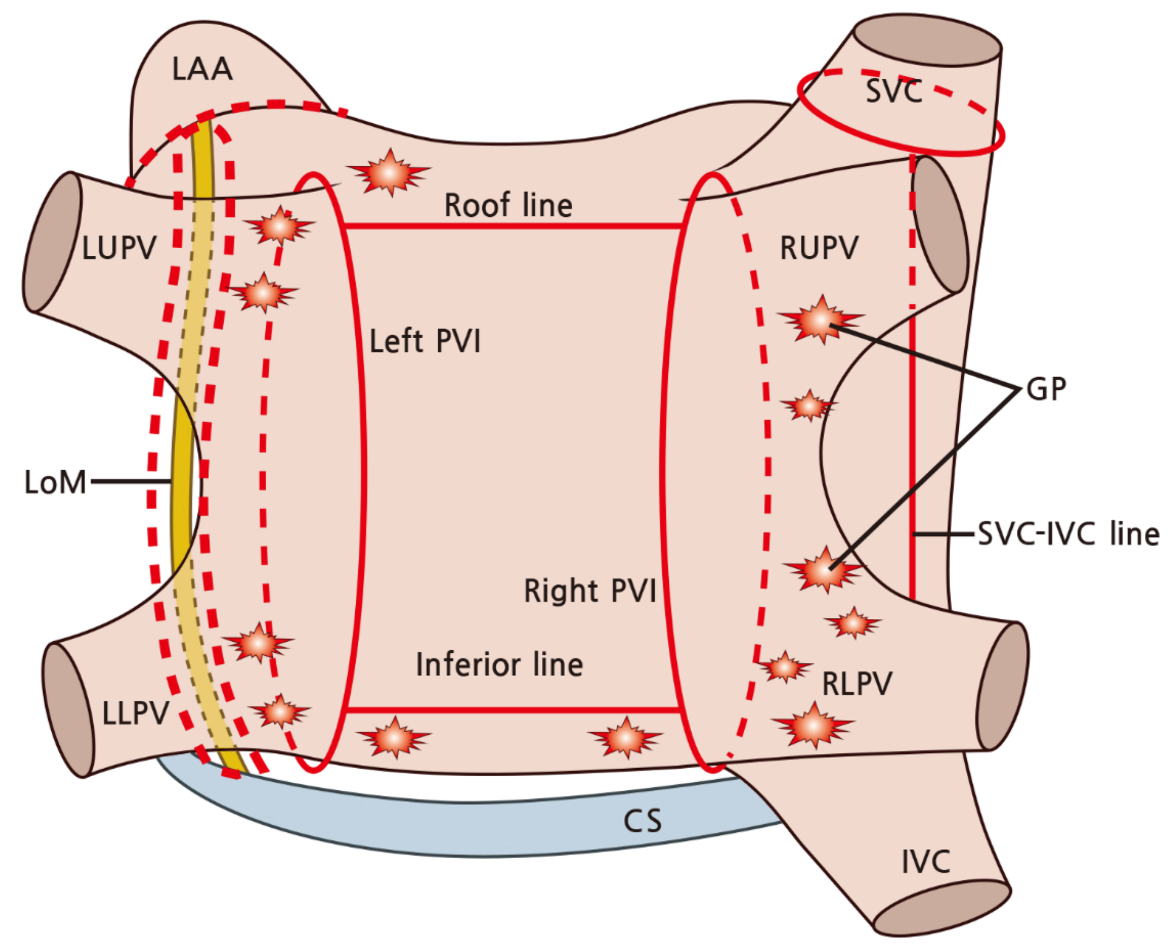



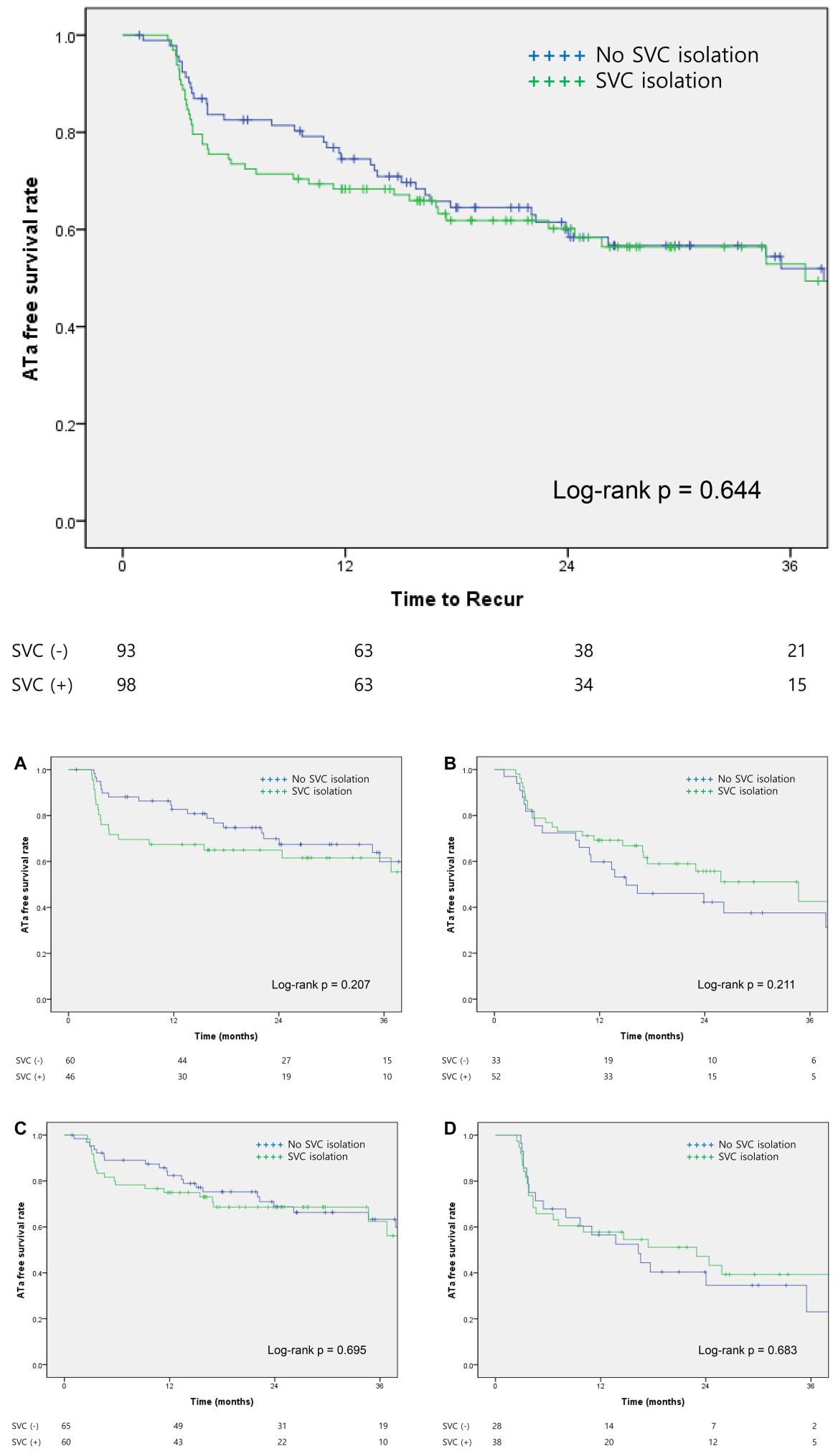